\title{
The influence of clumping on surface brightness fits of edge-on spiral galaxies
}

\author{
A. Misiriotis ${ }^{1,2}$ and S. Bianchi $i^{3,4,5,6}$ \\ 1 University of Crete, Physics Department, PO Box 2208, 71003 Heraklion, Creete, Greece \\ 2 Observatoire de Marseille, 2 place Le Verrier, 13248 Marseille Cedex 4, France \\ 3 Max-Planck-Institute für Astronomie, Königstuhl 17, 69117 Heidelberg, Germany \\ 4 European Southern Observatory, Karl-Schwarzschild-Strasse 2, 85748 Garching, Germany \\ 5 Max-Planck-Institute für Astrophysik, Karl-Schwarzschild-Strasse 1, 85741 Garching, Germany \\ ${ }^{6}$ CNR/Istituto di Radioastronomia - Sezione di Firenze, Largo E. Fermi 5, 50125 Firenze, Italy
}

Received 27 August 2001 / Accepted 16 January 2002

\begin{abstract}
We have used a Monte Carlo radiative transfer code to produce edge-on images of dusty galactic disks, allowing a fraction of the dust to be distributed in clumps. Synthetic images of edge-on galaxies have been constructed for different amounts of dust, distributions of clumps and fractions of dust in clumps, following the formalism of Bianchi et al. (2000a). We have also considered models with stellar emission embedded in the clumps. The synthetic images have been fitted with analytical models made with smooth distributions of dust, adopting the procedure developed by Xilouris et al. (1999) to fit optical images of real edge-on galaxies. We have compared the parameters determined by the fit with the input parameters of the models. For the clumping distributions adopted in this paper, the neglect of clumping results in underestimating the amount of dust in a galaxy. However, the underestimation is never larger than $40 \%$.
\end{abstract}

Key words. radiative transfer - ISM: dust, extinction - galaxies: photometry - galaxies: spiral - galaxies: structure

\section{Introduction}

Optical extinction by interstellar grains complicates the study of the stellar and dust content of a spiral galaxy. For moderate to high optical depths, information on the intrinsic properties of each of these components is entangled in the observed surface brightness distribution of the galaxy. The usual way of deriving these properties is by comparing the observations with radiative transfer calculations in a dusty galactic model. A certain degree of complexity is needed in the description of the geometric distributions of a galactic model. Radiative transfer models with simple geometries have been proven to provide equivocal results (Disney et al. 1989). Furthermore, scattering must be taken into account, given the high albedo observed for Galactic dust (Gordon et al. 1997).

A radiative transfer code using the Monte Carlo (MC) technique can, in principle, accommodate any stellar and dust distribution and provide an accurate calculation of the surface brightness for a given model (Witt et al. 1992; Bianchi et al. 1996; de Jong 1996). However, the MC method is time consuming and thus cannot be

Send offprint requests to: S. Bianchi, e-mail: sbianchi@arcetri.astro.it easily included in fitting procedures. For such procedures an approximate treatment of the radiative transfer is more appropriate.

The first approach of such a procedure was introduced by Kylafis \& Bahcall (1987, hereafter KB), where vertical profiles of a model galaxy were fitted to observations of NGC 891. In KB's work, scattering is calculated up to the first order and an approximation is introduced for the higher order contributions while the distribution of the dust is assumed to be smooth and exponential along the radial and vertical directions. A similar approximation for the treatment of scattering is adopted by Silva et al. (1998). Other works prefer an exact solution for scattering within simple geometries (Bruzual et al. 1988; Corradi et al. 1996; Xu \& Buat 1995).

Using the KB method, the radiative transfer equation can be solved for a wide variety of geometries (Byun et al. 1994). Xilouris et al. $(1997,1998)$ improved the original idea of $\mathrm{KB}$ and implemented a technique to fit the surface brightness distribution of edge-on spirals. In edge-on galaxies, the effects of extinction are maximized and it is possible to separate the stellar and dust components. The method has been successfully applied to a sample of seven edge-on galaxies (Xilouris et al. 1999) concluding 
that late-type spiral galaxies have a moderate opacity, with a mean face-on optical depth in the $B$-band $\tau_{B} \approx 0.8$. As in most of the models used for the description of spiral galaxies, Xilouris et al. (1999) adopt smooth exponential distributions to describe the stellar and dust disks.

Real galaxies exhibit a wide variety of inhomogeneities, like spiral arms, bars, clumps. While it is possible to include these structures in the solution, when fitting an observed image it is desirable to deal with the simplest possible description, in order to limit the number of model parameters. Complex models can then be used to test the reliability of the description obtained with the simple models. This is done, for example, in Misiriotis et al. (2000), where synthetic edge-on images of model galaxies with a spiral structure are fitted with a smooth exponential model. They find that plain exponential distributions provide a good description of the galactic disks. The derivation of the stellar and dust parameters is only slightly affected by the spiral pattern.

In this paper we perform a similar exercise to study the influence of dust clumping on the fit of edge-on galaxies. In most cases, a clumpy medium exhibits higher transparency than a smooth one of the same dust mass. Therefore, the comparison of real images with smooth models may result in an underestimation of the dust content in a galaxy. Here we use the MC method presented by Bianchi et al. (2000a, hereafter BFDA) to create images of clumpy galactic models. For the parametrisation of BFDA, the maximum difference between clumpy and homogeneous models can be seen in the edge-on case. Monte Carlo models of highly inclined galaxies had been produced also by Kuchinski et al. (1998), with a different parametrisation for the clump distribution. With respect to their result, the influence of clumping in the edge-on case is larger for the model adopted here (see the discussion in BFDA). The MC images of BFDA will be fitted with the KB model as in Misiriotis et al. (2000), aiming at determining the efficiency of clumping in hiding the dust when a galaxy is seen edge-on.

Section 2 describes the method adopted for the comparison and the MC models we have used to produce the synthetic images. A model with exponentially distributed clumps developed for this work is presented in the Appendix. The results are presented in Sect. 3 and a summary is given in Sect. 4 .

\section{Our method}

Following the procedure described by Misiriotis et al. (2000), we fit images made with the KB method to the MC images of a clumpy model galaxy. Both the MC and the $\mathrm{KB}$ models presented here are computed for the $V$-band. The Henyey \& Greenstein (1941) phase function is used with the scattering parameters in the $V$ band albedo $\omega=0.6$ and asymmetry $g=0.6$ (Gordon et al. 1997).

The parameters defining the smooth models are the central face-on surface brightness $I_{\star}$, the radial scalelength $\alpha_{\star}$ and the vertical scalelength $\beta_{\star}$ of the stellar disk, the
$V$-band central face-on optical depth $\tau_{V}$, the radial scalelength $\alpha_{\mathrm{d}}$ and the vertical scalelength $\beta_{\mathrm{d}}$ of the dust disk. The total intrinsic luminosity $L_{\star}$ is equal to $4 \pi I_{\star} \alpha_{\star}^{2}$ while the dust mass $M_{\mathrm{d}}$ is propotional to $\tau_{V} \alpha_{\mathrm{d}}^{2}$. The results from the fits are compared to the input parameters of the MC images and are analyzed as a function of the fraction of dust locked into clumps $f_{\mathrm{c}}$. Additionally, we study the importance of scattering in the fitted models.

The disk parameters adopted for the MC models are those used in BFDA. For the stellar disk, we have adopted a ratio between radial and vertical scalelengths $\alpha_{\star} / \beta_{\star}=$ 11.5. The smooth dust disk has the same radial scalelength as the stellar one and a vertical scalelength $\beta_{\mathrm{d}}=0.4 \beta_{\star}$. In both the simulation and the fitting procedure the dust distribution has been truncated at 4.6 radial scalelengths along the radial direction and at 4 vertical scalelengths along the vertical direction.

In the clumpy images, $\tau_{V}$ defines the total mass of dust in the model, i.e. for the same $\tau_{V}$ the clumpy and the smooth models have the same dust mass. In partially clumpy models, a fraction $f_{c}$ of the total dust mass is distributed in clumps, while the remaining is distributed in a smooth disk of central face-on optical depth $\tau_{V}\left(1-f_{\mathrm{c}}\right)$. The number of the clumps $N_{\mathrm{c}}$ is determined as described in BFDA.

To cover the full range of $\tau_{V}$ and $f_{\mathrm{c}}$ we considered the following optical depths: $\tau_{V}=0.5,1,2$ and 5. For each optical depth we produced images with $f_{\mathrm{c}}=0$ (smooth case), 0.25, 0.5, 0.75 and 1.0, resulting in a set of 20 images. Several different sets can be produced depending on how we distribute the dust clumps. We have decided to produce 3 sets.

In the set named "molecular", the dust clumps follow the same distribution as the molecular gas in the Galaxy and their characteristics are based on the Giant Molecular Clouds. Details on these models can be found in BFDA. In this set, the $f_{\mathrm{c}}=1$ case is not considered because it would mean that all the dust is distributed in a molecular ring and an exponential model is not recommended to reproduce such a distribution.

A second set of models named "exponential" was produced with the dust clumps following the same exponential distribution as the smooth dust disk while the stars were smoothly distributed. The exponential models are described in detail in the Appendix.

In the third set of models named "embedded" the clumps are distributed exponentially, while a fraction of starlight $f_{\mathrm{emb}}=0.5$ was embedded in the dust clumps. For the case of $f_{\mathrm{c}}=0$, there can be no $f_{\mathrm{emb}}=0.5$ because when there are no clumps there is nowhere to embed the embedded starlight.

The MC images of the clumpy model galaxies were constructed to be as similar as possible to observations. The images cover 151 pixels along the major axis from the galactic center to the border of the image, while the radial scalelength of the stellar and the dust disk is equal to 50 pixels. For each image, photons are gathered in a finite inclination band (Bianchi et al. 1996). which was chosen 


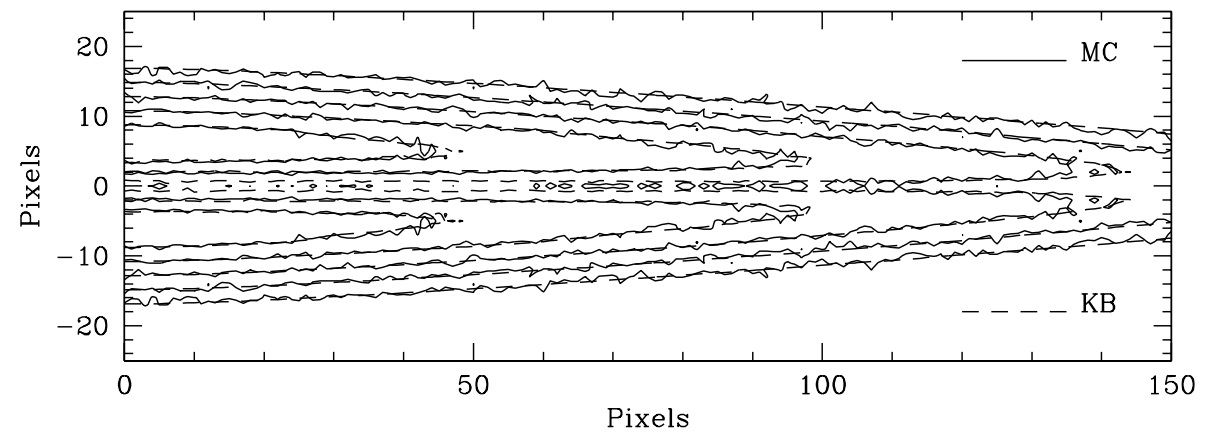

Fig. 1. Contour plot of a synthetic edge-on image from a Monte Carlo smooth model with $\tau_{V}=1$. Overplotted are isophotes from a Kylafis \& Bahcall (1987) type model.

to be $1^{\circ}$. The number of photons for the MC simulations was chosen so that a realistic signal to noise ratio was achieved (Bianchi et al. 1996).

The fitting was performed as in Misiriotis et al. (2000), by minimizing the sum of the squares of the differences between the $\mathrm{MC}$ and the $\mathrm{KB}$ images. In Fig. 1 we present a contour plot of an edge-on image from a MC smooth model $\left(f_{\mathrm{c}}=0\right)$ together with the isophotes of the same model image, produced using the KB method. The coincidence of the MC and KB model images demonstrates the consistency of the two methods.

\section{Results of the fits}

\subsection{Testing of the codes and the effects of scattering}

Since the two radiative transfer methods (BFDA and KB) used in this work have been developed independently and with different algorithms, we checked for their consistency in several ways. We first produced images without scattering and without clumps using the MC method and fitted them with the KB method. The fits were able to retrieve all the parameters with a scatter smaller than $4 \%$.

We then analyzed the smooth models $\left(f_{c}=0\right)$ described in the previous section. Two fits were produced for each MC image. In the first, the fitting model is computed without scattering, while in the second scattering is taken into account. This way we can quantify both the importance of scattering in fits of edge-on galaxies and the approximation of $\mathrm{KB}$ in their treatment of scattering.

In Fig. 2 we show the parameters $I_{\star}$ and $\tau_{V}$ whose behavior changes the most when passing from an absorptiononly fit to a fit including scattering. It is well-known that scattering reduces the effective absorption of a dusty medium, thus resulting in an increased surface brightness. When scattering is not included (dotted lines in Fig. 2), the fitting procedure compensates for this by reducing the optical depth of the dust disk and by increasing the central surface brightness of the stellar disk. The underestimate of the dust content is the dominant effect, because the stellar luminosity is significantly constrained by the parts of the galaxy that lie outside of the dust lane, where the absorption is negligible. Absorption-only fits result in an underestimate of the face-on optical depth ranging from $10 \%$ for $\tau_{V}=0.5$ to $25 \%$ for $\tau_{V}=5$. As for the central

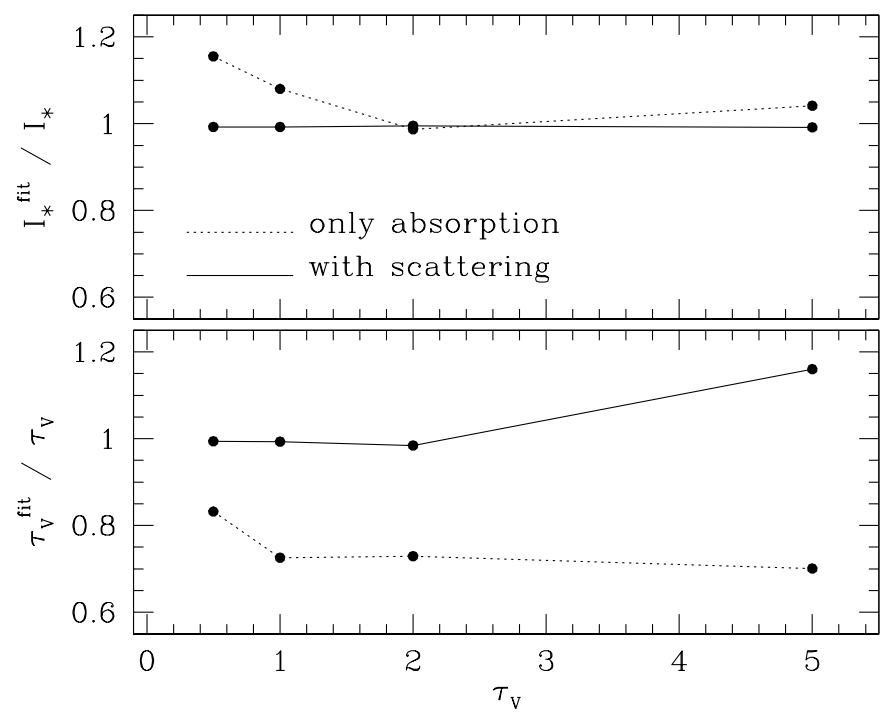

Fig. 2. The derived $I_{\star}$ and $\tau_{V}$ normalized to the input values as functions of the optical depth of the model. Results for fits with and without scattering are shown.

face-on surface brightness $\left(I_{\star}\right)$, the maximum discrepancy is an overestimation by $10 \%$ for $\tau_{V}=0.5$

When scattering is included, the fitted parameters are very close to the input values (solid lines in Fig. 2). The $\tau_{V}$ plot confirms the validity of the approximation for scattering introduced by Kylafis \& Bahcall (1987), at least for low-to-moderate optical depths. Even for $\tau_{V}=5$, the derived optical depth is only $15 \%$ larger than the input value. All the other parameters derived from fits with scattering were found to vary within less than $4 \%$ of the input values.

\subsection{The effect of clumping}

The quality of the fits is poorer when synthetic images from clumpy models are analyzed. The sum of the squares of the differences between input data and fitted models increases by about an order of magnitude. Furthermore, in many cases it was possible to find a few equally acceptable fits, corresponding to local minima. While some parameters of KB models may change from minimum to minimum, we found that the derived dust mass and intrinsic luminosity are nearly the same.

In Fig. 3 we have plotted the derived normalized dust mass and intrinsic luminosity, as functions of $f_{\mathrm{c}}$ and of $\tau_{V}$, 


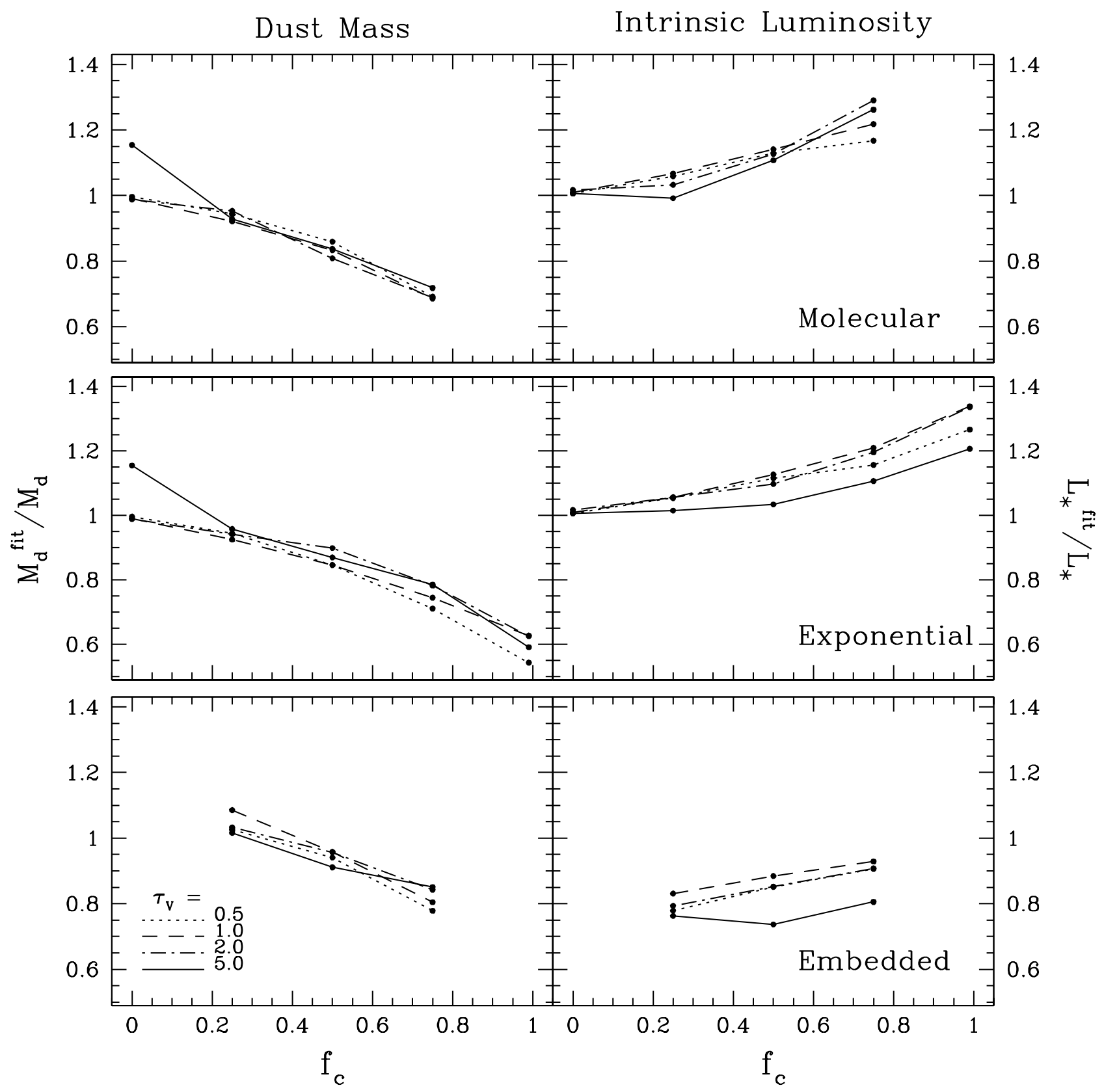

Fig. 3. Normalized dust mass (left column) and normalized intrinsic luminosity (right column) derived by fitting the Monte Carlo simulations of Sect. 2, as functions of the fraction $f_{\mathrm{c}}$ of dust locked up in clumps. The values have been normalized to the input dust mass and intrinsic luminosity. Results are presented for models with optical depths $\tau_{V}=0.5$ (dotted lines), 1 (dashed), 2 (dot-dashed) and 5 (solid). The plots in the top row show the results for the clumpy model of BFDA with fraction of embedded stellar emission $f_{\text {emb }}=0$; the middle row refers to the models with clumps distributed exponentially and $f_{\text {emb }}=$ 0 ; the bottom row to exponentially distributed clumps, but with $f_{\text {emb }}=0.5$. Results for the smooth models $\left(f_{\mathrm{c}}=0\right)$ are plotted in both the top and middle row.

for the "molecular" models, (BFDA; top row); for the "exponential" models (middle row) and for the "embedded" models (bottom row).

The derived dust masses, normalized to the input values, are shown in the left column of Fig. 3. The trend of the dust mass with $f_{\mathrm{c}}$ does not depend on the type of clumping or on the optical depth of the model, the amount of detected dust decreases with increasing $f_{\mathrm{c}}$. For the two sets of models without embedded stellar emission ("molecular" and "exponential"), the dust mass is underestimated by less than $10 \%$ for $f_{\mathrm{c}}=0.25$ to about $20-30 \%$ for $f_{\mathrm{c}}=0.75$. For the "exponential" set for $f_{\mathrm{c}}=1$, the underestimate reaches values of $40 \%$. For the "embedded" models the fitting procedure recovers the input dust quite well. All the dust is detected for $f_{\mathrm{c}}=0.25$ and only $20 \%$ is missed for $f_{\mathrm{c}}=0.75$.

The results of the fitting procedure for the intrinsic, dust-free, luminosity $L_{\star}$ are shown in the right column of 
Fig. 3, again normalized to the input value. As for the dust mass, the trends with $f_{\mathrm{c}}$ for different models are very similar and do not depend sensitively on the value of $\tau_{V}$. The fitting model overestimates the luminosity with increasing $f_{\mathrm{c}}$. For $f_{\mathrm{c}}=0.75$ and $f_{\mathrm{emb}}=0$, the derived luminosity is about $20 \%$ larger than the input value.

The overestimate of the luminosity drives the fitting procedure to increase the dust mass in order to obscure the additional starlight. If the luminosity was not overestimated then even less dust would be needed to fit the synthetic images. A simple major-axis analysis, i.e. a comparison of the radial profiles along the dust lane suggests that the smooth model detects only half of the dust mass (BFDA) and leads to the conclusion that clumping causes an underestimate of the face-on optical depth.

This is not the case for the fits shown here. The fitting procedure tries to interpret the reduced extinction caused by clumping by decreasing the dust radial scalelength $\alpha_{d}$ and keeping the optical depth high (actually, to a value similar to the input one), rather than decreasing $\tau_{V}$ and keeping $\alpha_{\mathrm{d}}$ fixed. The reason for this may be that the surface brightness in models with clumping departs from that of a smooth exponential disk, when all the image, and not only a single profile, is fitted. Given the general trend of the retrieved data, it does not seem probable that we have systematically hit local minima of the fit.

We have shown that the trend of dust mass underestimation is nearly independent of the optical depth of the model. This may suggest that the same percentage of dust is missed when fitting a galaxy at different wavelengths. Therefore, clumping should not affect the determination of the extinction law from fits of a galaxy in different bands. As long as the dust properties do not vary significantly with wavelength, as in the optical range, it is possible to interpret the absence of a trend with $\tau_{V}$ as an absence of a trend with $\lambda$.

\section{Summary}

We have used a Monte Carlo code for the radiative transfer in a clumpy galactic model to produce synthetic edgeon images of spiral galaxies in the $V$-band. The images have then been analyzed using the fitting procedure of Xilouris et al. (1999), that uses the method of Kylafis \& Bahcall (1987) to compute the radiative transfer in smooth galactic models. We first checked for the consistency of the two independent codes, by analyzing smooth MC images. Despite the differences in the solution of the radiative transfer, it was possible to retrieve the parameters of the MC models within $4 \%$ of their original value and we confirmed the importance of scattering in the radiative transfer simulations. The dust mass is significantly underestimated when scattering is not included in the fitting routine.

We have then studied the influence of clumping on the derivation of the parameters, when a synthetic image of an edge-on clumpy galactic model is analyzed through a simpler (and more controllable) smooth model.
As expected, using a smooth model when fitting images of edge-on galaxies leads to an underestimation of the dust content of the galaxy. For the distribution of clumps adopted in this paper, about $20-30 \%$ of the dust mass is missed by the fitting, the value depending more on the fraction of dust located in clumps rather than on the details of the clumps distribution or the total dust mass of the model. In addition, clumping alters the surface brightness distribution with respect to that of a smooth exponential model, thus making the fit more difficult. This may explain the overestimation of the intrinsic luminosity in models with clumping.

We stress again here that our work is limited to the edge-on case, for which convergent fits can be obtained with the KB technique. The results may also depend on the clump distribution and on the geometrical parameters adopted here. However, it is interesting to note that other works find a small influence of clumping in edge-on galaxies. Kuchinski et al. (1998) produced radiative transfer models of disk galaxies including clumping. The distribution of clumps is different from the one adopted here: the clumpy medium is characterised by a costant filling factor throughout the whole disk and by a density contrast with the homogeneous medium (Witt \& Gordon 1996, see also BFDA for a comparison with the present models). Comparing colour gradients across the extinction lane of highly inclined galaxies with the model results, they find that the inferred opacity of the dust disk is largely insensitive to the difference between clumpy and homogeneous dust distributions.

Recent analyses of dust emission at $\lambda>100 \mu \mathrm{m}$ suggest that the amount of dust in spiral galaxies is larger than what derived from fits of edge-on surface brightness profiles (Bianchi et al. 2000b; Popescu et al. 2000; Misiriotis et al. 2001). Clumping has been imputed to as a possible cause for this discrepancy: dust in starforming regions may contribute dominantly to the Far Infrared emission without being seen through its extinction effects. However, the underestimation produced by the clumpy distributions adopted in this paper is always smaller than $40 \%$, unable to explain the magnitude of the effect. Different dust/clump distributions may be needed. Popescu et al. (2000) and Misiriotis et al. (2001) find that the long wavelength emission observed in two galaxies (namely, NGC 891 and NGC 5907) can be explained by including a second dust disk, at least as massive as the dust disk causing the observed extinction lane and thin enough to escape detection through the technique of surface brightness fitting.

Acknowledgements. We would like to thank Professor N. D. Kylafis for his suggestions and assistance concerning this work, and the referee, Professor A. Witt, for thoughtful comments.

\section{Appendix A: Models with exponentially distributed clumps}

In BFDA it was assumed that all the material associated with the molecular phase in a spiral galaxy is in Giant 


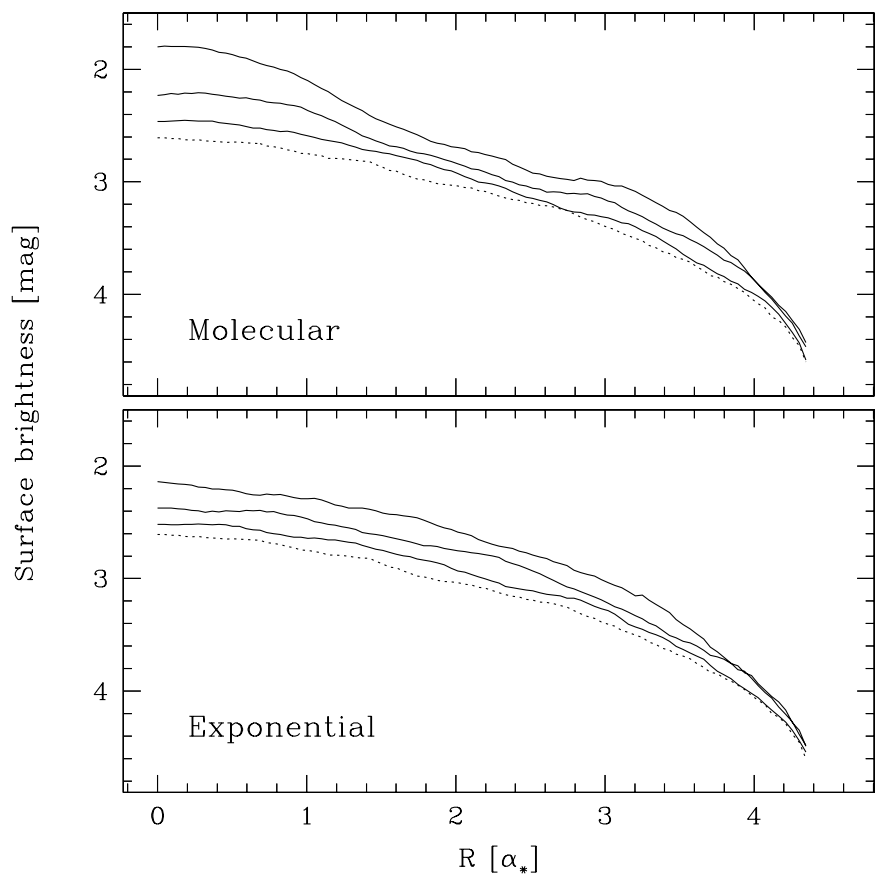

Fig. A.1. Major-axis edge-on profiles for $\tau_{V}=2$ models with molecular (top panel) and exponential (bottom panel) clumping, for $f_{\mathrm{c}}=0.25,0.5$ and 0.75 (from bottom to top, solid lines). As a reference, we also show the profile for a $\tau_{V}=2$ smooth model (dotted lines).

Molecular Clouds. Radiative transfer models were produced assuming that dust clumps are distributed as the molecular gas in the Galaxy, i.e. in a ring-like structure. However, most of luminous, face-on, late-type galaxies, and a good fraction of early-type spirals, show a radial exponential distribution for the $\mathrm{H}_{2}$ column density (Young \& Scoville 1991). For the sake of completeness, we briefly present here a set of models with an exponential distribution for the dust clumps. The clumps distribution has the same scalelengths as the smooth dust disk. All the other parameters are the same as in BFDA.

The effects of clumping in these models are not very different from those obtained adopting the Galactic $\mathrm{H}_{2}$ distribution. Exponentially distributed clumps make the models less transparent, but only slightly. For example, in the $\tau_{V}=2$ model with fraction of gas in clumps $f_{\mathrm{c}}=$ $0.75,15 \%$ of the radiation is absorbed, while in BFDA it was $12 \%$. We recall that in a smooth model $19 \%$ of the radiation is absorbed. For models with embedded stellar emission, the difference is even smaller, because of the increased contribution of the absorption within each clump.
The number of clumps and their opacity are independent of the assumed clump distribution and are the same as in BFDA.

As in BFDA, the largest differences between homogeneous and clumpy simulations are for the edge-on profiles. In Fig. A.1 we show the edge-on major-axis profiles for the BFDA models and for the models with exponential clumping, for $\tau_{V}=2, f_{\mathrm{c}}=0.25,0.5,0.75$ and $f_{\mathrm{emb}}=0$. The profile for a smooth $\tau_{V}=2$ disk is shown as a reference. Because of the exponential distribution, the profiles of the new clumpy models are similar to those for smooth exponential disks, independently of $f_{\mathrm{c}}$ and $f_{\mathrm{emb}}$. Instead, BFDA models show the effects of the ring-like clumping distribution, even when $f_{\mathrm{emb}}=0$.

\section{References}

Bianchi, S., Ferrara, A., Davies, J. I., \& Alton, P. B. 2000a, MNRAS, 311, 601

Bianchi, S., Davies, J. I., \& Alton, P. B. 2000b, A\&A, 359, 65

Bianchi, S., Ferrara, A., \& Giovanardi, C. 1996, ApJ, 465, 127

Bruzual, A. G., Magris, G., \& Calvet, N. 1988, ApJ, 333, 673

Byun, Y. I., Freeman, K. C., \& Kylafis, N. D. 1994, ApJ, 432, 114

Corradi, R. L. M., Beckman, J. E., \& Simonneau, E. 1996, MNRAS, 282, 1005

de Jong, R. 1996, A\&A, 313, 377

Disney, M., Davies, J., \& Phillipps, S. 1989, MNRAS, 239, 939

Gordon, K. D., Calzetti, D., \& Witt, A. N. 1997, ApJ, 487, 625

Henyey, L. G., \& Greenstein, J. L. 1941, ApJ, 93, 70

Kuchinski, L. E., Terndrup, D. M., Gordon, K. D., \& Witt, A. N. 1998, AJ, 115, 1438

Kylafis, N. D., \& Bahcall, J. N. 1987, ApJ, 317, 637

Misiriotis, A., Kylafis, N. D., Papamastorakis, J., \& Xilouris, E. M. 2000, A\&A, 353, 117

Misiriotis, A., Popescu, C. C., Tuffs, R. J., \& Kylafis, N. D. 2001, A\&A, 372, 775

Popescu, C. C., Misiriotis, A., Kylafis, N. D., Tuffs, R. J., \& Fischera, J. 2000, A\&A, 362, 138

Silva, L., Granato, G. L., Bressan, A., \& Danese, L. 1998, ApJ, 509, 103

Witt, A. N., \& Gordon, K. D. 1996, ApJ, 463, 681

Witt, A. N., Thronson, Harley, A. J., \& Capuano, J. M. J. 1992, ApJ, 393, 611

Xilouris, E. M., Kylafis, N. D., Papamastorakis, J., Paleologou, E. V., \& Haerendel, G. 1997, A\&A, 325, 135

Xilouris, E. M., Alton, P. B., Davies, J. I., et al. 1998, A\&A, 331, 894

Xilouris, E. M., Byun, Y. I., Kylafis, N. D., Paleologou, E. V., \& Papamastorakis, J. 1999, A\&A, 344, 868

Xu, C., \& Buat, V. 1995, A\&A, 293, L65

Young, J. S., \& Scoville, N. Z. 1991, ARA\&A, 29, 581 